\section{Prehospital tracheotomy in a case of avulsion of the larynx with a comminuted fracture of the jawbone}

\author{
Holger Rupprecht, Katharina Gaab \\ Surgical Department, Clinical Center Fuerth, Fuerth, Germany
}

Emergency physicians in the field are sometimes confronted with cases wherein patients cannot be intubated and ventilated. In some cases, cricothyrotomy, the method of choice for securing an emergency airway, may not have a successful outcome. We report a rare case of a 35-yearold male patient with avulsion of the larynx and a comminuted fracture of the jawbone, due to entrapment in a dung excavator. Prehospital tracheotomy was successfully performed. In cases with crush injuries to the larynx, anatomic structures, including the ligamentum conicum, are destroyed. In addition, massive subcutaneous emphysema blurs the anatomical key structures; hence, only a tracheotomy can prevent a lethal outcome.

Keywords Fractures, cartilage; Emergency medical services; Tracheotomy
eISSN: 2383-4625

Received: 30 March 2018

Revised: 5 April 2018

Accepted: 6 April 2018

Correspondence to: Katharina Gaab Surgical Department, Clinical Center Fuerth, Jakob-Henle-Straße 1, Fuerth 90766, Germany

E-mail:katharina@gaab-web.de ORCID

http://orcid.org/0000-0001-7464-3509

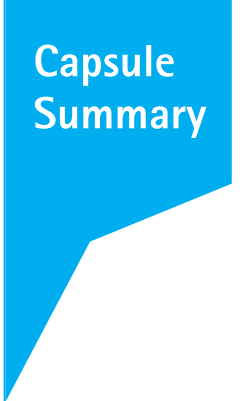

What is already known

Prehospital cricothyrotomy for securing an airway is usually recommended in emergency situations.

What is new in the current study

Prehospital tracheotomy is rarely performed, but in some cases where all other methods fail, it is the only choice for securing an airway. 


\section{INTRODUCTION}

Emergency physicians in the field are sometimes confronted with patients who cannot be intubated and ventilated. Moreover, in some cases, cricothyrotomy, the method of choice for securing an emergency airway may not have a successful outcome. In the literature, few cases of prehospital tracheotomy have been reported previously. ${ }^{1-5}$

\section{CASE REPORT}

We describe the case of a 35-year-old male patient whose head became entrapped in a dung excavator, which is an agronomical device. The patient was able to disentangle himself from the object. On the arrival of emergency medical service at the scene, the patient was leaning against a wall and expectorating blood and fragments of teeth and bones.

Cervical subcutaneous emphysema was conspicuous and increasing constantly. The patient was aphonic but approachable. He was questioned about the presence of severe pain and responded with affirmative nodding of the head. The patient declined supine positioning and wanted to sit upright.

During placement of intravenous access using a 14-gauge needle acute airway obstruction occurred. The patient became cyanotic with a decrease in oxygen saturation to $80 \%$; and began to strike out wildly in fear of death. After rapid injection of 50-mg ketamine and 20-mg etomidate, attempted oral intubation failed. The glottis was not adjustable because of the massive bleeding and fragments from the dislocated jawbone involving fracture of the maxilla and mandible. Suctioning of the airway brought up

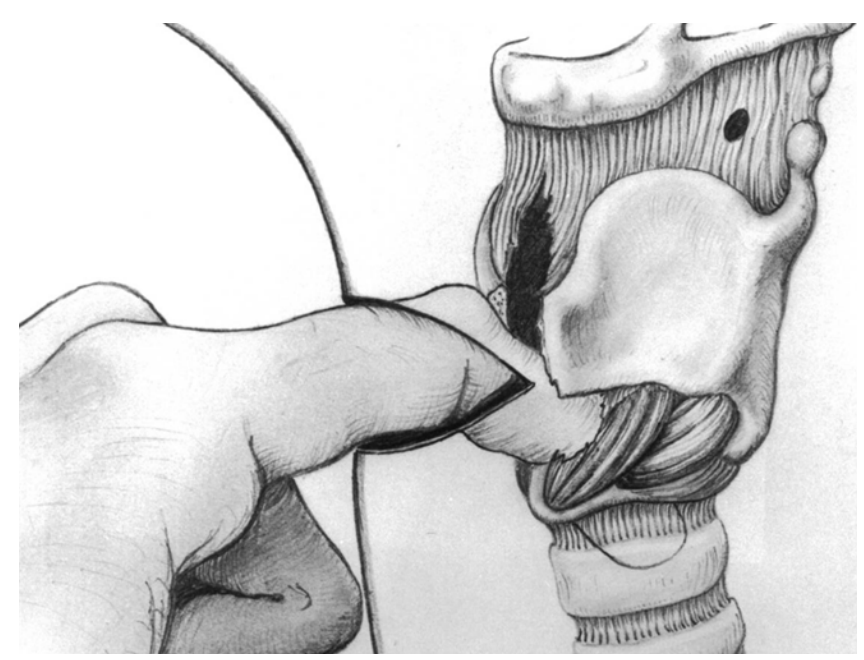

Fig. 1. Anatomical diagram showing the region of trauma and location of surgical intervention. several cartilaginous particles.

Due to the 'cannot intubate, cannot ventilate' situation and bradycardia of $<50$ beats per minute, an immediate cross-cervical skin incision was made with a scalpel according to the Kocher transverse collar incision method (2 fingerbreadths proximal to the jugular fossa). A large volume of bloody-foamy secretions from the tissue was noted. Subsequently, the partly destroyed straight cervical muscles were spread open and the trachea was bluntly exposed. This was done by blunt manual preparation, as well as by sharp preparation by means of a scissor. The dislocated, shattered larynx, which had shifted under the left jaw angle, was exposed. The tracheal defect was stretched manually (Fig. 1), in order to insert a 6.0-tube with cuff; blood that appeared lightred was aspirated (Fig. 2).

After ventilation with 100\% oxygen, the peripheral oxygen saturation showed an increase to normal levels within a minute; in addition, the heart rate increased to 100 beats per minute. After securing of the airway, analgosedation was performed with $0.5-\mathrm{mg}$ fentanyl and 10-mg diazepam. After placement of a second intravenous access, 1-L Ringer's solution was administered.

The patient was then transported to the nearest hospital to exclude possible pneumothorax. Subsequently, the patient maintained stable vital signs (blood pressure, 140/80 $\mathrm{mmHg}$; pulse rate, 100/min) and could then be transported to the University clinic (otorhinolaryngology) by an emergency rescue helicopter. Results from the computed tomography scan indicated a dislocated thyroid cartilage with the tube that was inserted prehospitally (Fig. 3), a mandibular fracture with soft tissue emphysema (Fig. 4), as well as a maxillary fracture.

On the same day, the patient underwent reconstruction of the

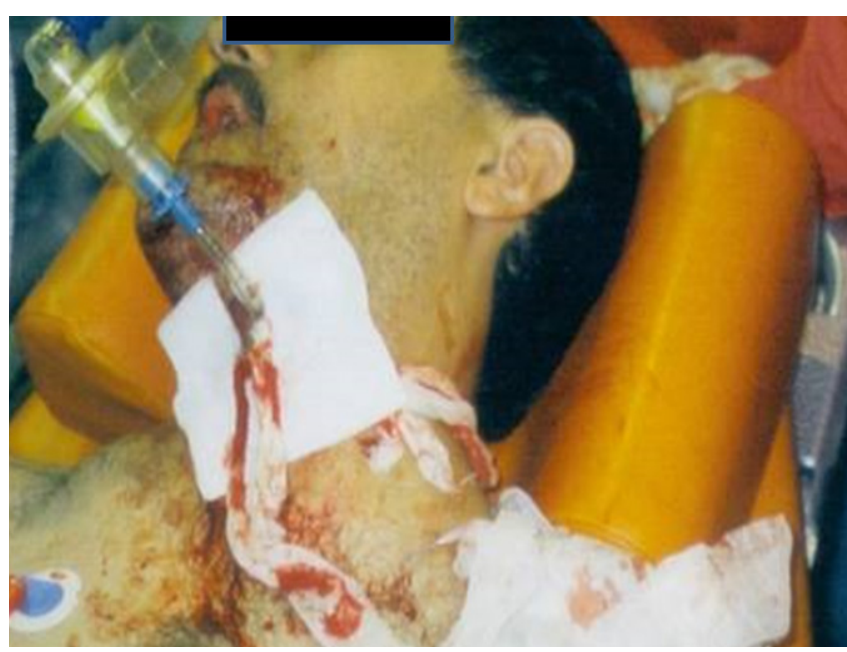

Fig. 2. Patient after insertion of a 6.0-tube with cuff. The participant gave informed consent to the publication. 


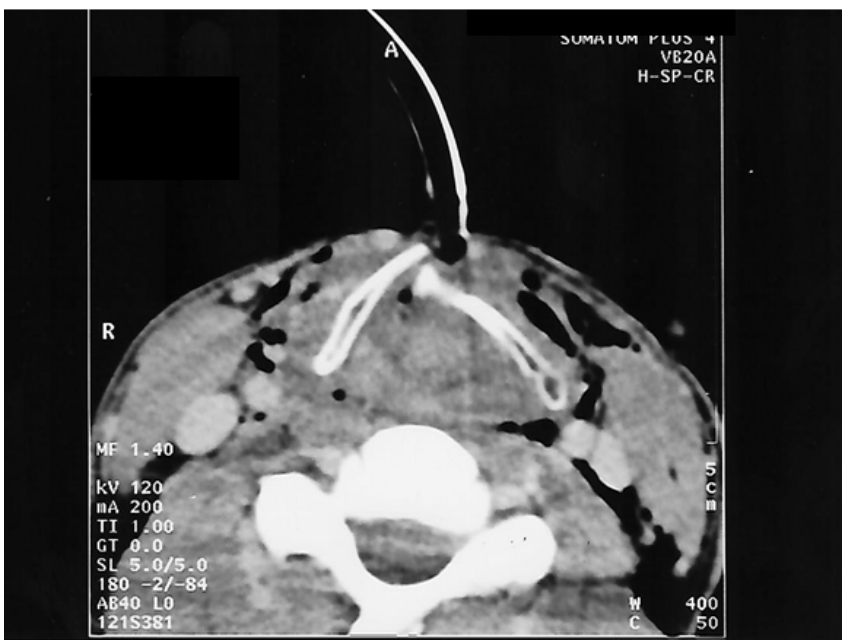

Fig. 3. Dislocated thyroid cartilage with the tube inserted prehospitally.

larynx (micro-plate osteosynthesis of the thyroid cartilage lamina) followed by osteosynthetic treatment of the fractured maxilla and mandible. A definitive tracheotomy was also performed.

Follow-up examination revealed the absence of neurological deficits, and only a slight limitation in the phonetic quality of speech. Currently, the patient has resumed work on the farm.

\section{DISCUSSION}

Fractures of the larynx are rare injuries with an incidence rate of one in 30,000 because the larynx is relatively protected by the sternum and the mandible. ${ }^{1-4}$ Typically, the massive traumatic impact required to cause laryngeal fracture usually results in additional injuries such as midfacial fractures, fractures of the body of cervical vertebra, and craniocerebral trauma; in addition, the patients commonly develop pneumothorax. ${ }^{2,45}$ Overall, the patient population shows a high rate of mortality. ${ }^{2,4}$ The presenting symptoms include dyspnea, hoarseness, aphonia, hemoptysis, and pain during swallowing; massive cervical subcutaneous emphysema, which is frequently progressive, is the most apparent characteristic. ${ }^{2-4}$ The patient often only tolerates a seated position. ${ }^{1,3}$ In the presence of a 'cannot intubate' situation, i.e., invisible glottis, such as in cases of massive bleeding, an endotracheal intubation should not be forced, because the manipulation of the fragments of bones or cartilages can lead to complete obstruction and asphyxiation. ${ }^{1,2,4,5}$

If the patient shows spontaneous breathing on arrival of the emergency medical service, the prognosis includes the likelihood of survival. Oxygen should be given through a mask placed in front of the patient's mouth; however, not administered into the mouth, to avoid aspiration. ${ }^{1}$ Medication that causes depression of respi-

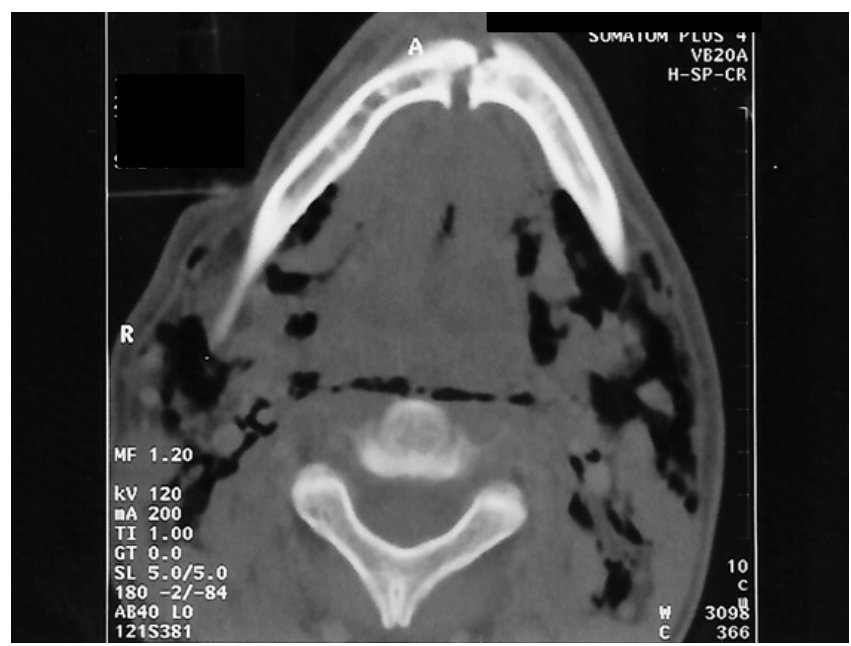

Fig. 4. CT showing mandibular symphysis fracture with soft tissue emphysema.

ration, especially opiates, should be delayed until after the airway is secured. Ketamine is the medication of choice, because it does not cause respiratory depression, protects airway reflexes, and does not cause hemodynamic instability at pain-relieving doses. ${ }^{1.4}$ For the skin incision, local anesthetics (lidocaine 1\%) should be injected. ${ }^{1.4,5}$

The cricothyrotomy, considered as the standard method of choice for securing an emergency surgical airway, is only indicated in cases with facial or jawbone fractures, as well as in cases of anaphylaxis with massive edema of the face. In cases of massive trauma to the larynx, the anatomic structures including the cricothyroid ligament are destroyed. The massive subcutaneous emphysema further blurs the anatomical key structures (e.g., laryngeal prominence), such that only a tracheotomy can prevent a lethal outcome. ${ }^{1,2,4,5} \mathrm{~A}$ sufficient cross-cervical incision is made two fingerbreadths proximal to the jugular fossa, with cross-incision of the platysma muscle as done for thyroidectomy. The injured cervical muscles are spread apart bluntly. Preparation should be performed at the center line to avoid additional bleeding, for example, through a lesion made in the thyroid gland. ${ }^{1,3,4}$ In our case, at the height of the 2nd to 3 rd tracheal ring, a ventral incision was made by means of a scalpel, through which, a 6-0 tube could be inserted. After securing the airway, analgosedation using fentan$\mathrm{y} /$ /midazolam or anesthesia was performed. ${ }^{1,4}$ The cervical wound or the oral cavity were treated additionally with compression dressings to control bleeding.

Orotracheal intubation is the primary method of securing an emergency airway. Fiberoptic-assisted nasotracheal intubation has gained popularity for managing difficult airways. The use of laryngeal mask airway and esophageal-tracheal combination tube is a temporary measure to provide ventilation until a definitive 
airway is obtained. In cases with an airway obstruction in which all non-surgical methods fail, cricothyroidotomy is the preferred technique; however, similar to our case, the larynx and the cricothyroid ligament are destroyed in such cases. In addition, the massive subcutaneous emphysema blurs the anatomical key structures; hence, only a tracheotomy can prevent a lethal outcome.

This case report highlights successful prehospital tracheotomy performed using a similar method as that for thyroidectomy, in a patient with avulsion of the larynx with a comminuted fracture of the jawbone.

\section{CONFLICT OF INTEREST}

No potential conflict of interest relevant to this article was reported.

\section{REFERENCES}

1. Bonanno FG. Issues of critical airway management (Which anesthesia; which surgical airway?). J Emerg Trauma Shock 2012;5:279-84.

2. Hurford $W E$, Peralta R. Management of tracheal trauma. Can J Anaesth 2003;50:R17-22.

3. Oh JH, Min HS, Park TU, Lee SJ, Kim SE. Isolated cricoid fracture associated with blunt neck trauma. Emerg Med J 2007; 24:505-6.

4. Ye D, Shen Z, Zhang Y, Qiu S, Kang C. Clinical features and management of closed injury of the cervical trachea due to blunt trauma. Scand J Trauma Resusc Emerg Med 2013;21:60.

5. Patel SA, Meyer TK. Surgical airway. Int J Crit IIIn Inj Sci 2014; 4:71-6. 